\title{
Spectra of finitely presented lattice-ordered Abelian groups and MV-algebras, part 1.
}

\author{
Vincenzo Marra ${ }^{1}$, Daniel McNeill ${ }^{2}$, and Andrea Pedrini1 ${ }^{1 *}$ \\ 1 Dipartimento di Matematica "Federigo Enriques" \\ Università degli Studi di Milano \\ Via Cesare Saldini 50, 20133 Milano, Italy \\ vincenzo.marra@unimi.it, andrea.pedrini@unimi.it \\ 2 Dipartimento di Scienze Teoriche e Applicate \\ Università degli Studi dell'Insubria \\ Via Mazzini, 5, 21100 Varese, Italy \\ danmcne@gmail.com
}

\section{Introduction}

This is the first part of a series of two abstract, the second one being by Daniel McNeill.

If $X$ is any topological space, its collection of opens sets $\mathscr{O}(X)$ is a complete distributive lattice, with joins given by unions, and meets given by $\bigwedge_{U \in S} U:=\operatorname{int}\left(\bigcap_{U \in S} U\right)$, for any $S \subseteq \mathscr{O}(X)$. Moreover, $\mathscr{O}(X)$ is a frame, i.e. it satisfies the frame law

$$
U \wedge\left(\bigvee_{V \in S} V\right)=\bigvee_{V \in S}(U \wedge V), \text { for any } S \subseteq \mathscr{O}(X), U \in \mathscr{O}(X)
$$

Thus, $\mathscr{O}(X)$ is also a Heyting algebra whose implication is defined as

$$
U \rightarrow V:=\bigvee\{Z \mid U \wedge Z \subseteq V\}, \text { for any } U, V \in \mathscr{O}(X)
$$

(See e.g. 6] for background.) When $X$ is equipped with a distinguished basis $D$ for its topology, closed under finite meets and joins, one can investigate situations where $D$ is also closed under the implication (2), i.e., where $D$ is a Heyting subalgebra of $\mathscr{O}(X)$.

Recall that $X$ is a spectral space if it is compact and $T_{0}$, its collection $D$ of compact open subsets forms a basis which is closed under finite intersections and unions, and $X$ is sober: any closed set that cannot be written as the union of two proper closed subsets, has a dense point. (In this case, the latter point is unique, because $X$ is $T_{0}$.) By Stone duality, spectral spaces are precisely the spaces arising as sets of prime ideals of some distributive lattice, topologised with the Stone or hull-kernel topology. Specifically, given such a spectral space $X$, its collection of compact open sets $D$ is (naturally isomorphic to) the distributive lattice dual to $X$ under Stone duality. We are going to exhibit a significant class of such spaces for which $D$ is a Heyting subalgebra of $\mathscr{O}(X)$. We work with lattice-ordered Abelian groups and vector spaces. Using Mundici's $\Gamma$-functor [8] the results can be rephrased in terms of $M V$-algebras, the algebraic semantics of Łukasiewicz infinite-valued propositional logic [5].

Recall that a lattice-ordered Abelian group [4, or $\ell$-group for short, is an Abelian group which is also a lattice, and is such that the group operation distributes over both meets and joins. Similary, a vector lattice (also known as a Riesz space), is a lattice-ordered real vector

*Presenting author. 
space such that addition distributes over meets and joins, and multiplication by non-negative real scalars also distributes over meets and joins. A $\mathbb{Q}$-vector lattice is defined analogously replacing real vector spaces with rational vector spaces. The classes of $\ell$-groups, vector lattices, and $\mathbb{Q}$-vector lattices are varieties of algebras. Hence free objects in each class exist, and finitely presented objects are standardly defined as quotients of finitely generated free objects modulo a finitely generated congruence. Further, since each structure in question has an obvious distributive-lattice reduct, each of the three varieties is congruence-distributive. It is known that the complete distributive algebraic lattice $\operatorname{Con} G$ of the congruences 11 of an $\ell$-group (or vector lattice, or $\mathbb{Q}$-vector lattice) $G$ is in fact relatively pseudo-complemented, i.e. is a Heyting algebra. We write $\mathrm{K}(G)$ for the subset of Con $G$ consisting of the finitely generated congruences of $G$. It can be shown that when $G$ is finitely presented, then $\mathrm{K}(G)$ is a sublattice of $\operatorname{Con} G$; see Section 2 for more details on this.

Theorem 1.1. Let $G$ be a finitely presented $\ell$-group (or vector lattice, or $\mathbb{Q}$-vector lattice), and let $\operatorname{Con} G$ be the Heyting algebra consisting of the congruences on $G$. Then the congruence

$$
\theta \rightarrow \eta:=\bigvee\{\zeta \mid \theta \wedge \zeta \subseteq \eta\}
$$

is finitely generated whenever $\theta, \eta \in \mathrm{K}(G)$. Equivalently, $\mathrm{K}(G)$ is a Heyting subalgebra of Con $G$.

\section{The Spectral Space of $G$}

Let $G$ be an $\ell$-group (or a vector lattice, or a $\mathbb{Q}$-vector lattice). A congruence $\theta$ on $G$ is proper if $\theta \neq G \times G$, prime if the quotient $G / \theta$ is totally ordered, and maximal if $\theta$ is proper and whenever $\eta \supseteq \theta$ is a congruence on $G$, then either $\eta=\theta$ or $\eta=G \times G$.

Let Spec $G$ be the set of all prime congruences of $G$. We define two maps $\mathbb{V}: 2^{G} \rightarrow 2^{\operatorname{Spec} G}$ and I: $2^{\operatorname{Spec} G} \rightarrow 2^{G}$ by setting, for each $S \subseteq G$ and for each $E \subseteq \operatorname{Spec} G$,

$$
\mathbb{V}(S):=\{\mathfrak{p} \in \operatorname{Spec} G \mid(s, 0) \in \mathfrak{p} \text { for all } s \in S\} \text { and } \mathbb{I}(E):=\{g \in G \mid(g, 0) \in \mathfrak{p} \text { for all } \mathfrak{p} \in E\} .
$$

The pair $(\mathbb{I}, \mathbb{V})$ forms a (contravariant) Galois connection: for all $S \subseteq G$ and $E \subseteq \operatorname{Spec} G$, $S \subseteq \mathbb{I}(E)$ if and only if $E \subseteq \mathbb{V}(S)$. This ensures that the maps $\mathbb{I} \circ \mathbb{V}: 2^{G} \rightarrow 2^{G}$ and $\mathbb{V} \circ \mathbb{I}: 2^{\operatorname{Spec} G} \rightarrow 2^{\operatorname{Spec} G}$ are closure operators. In particular, $\mathbb{I} \circ \mathbb{V}: 2^{G} \rightarrow 2^{G}$ is algebraic, i.e. it satisfies $\mathbb{I} \circ \mathbb{V}(S)=\bigcup \mathbb{I} \circ \mathbb{V}(F)$, as $F$ ranges over the finite subsets of $S$. Moreover, it can be shown that $\mathbb{V} \circ \mathbb{I}: 2^{\operatorname{Spec} G} \rightarrow 2^{\operatorname{Spec} G}$ is topological, i.e. it commutes with finite unions. Thus, the operator $\mathbb{V} \circ \mathbb{I}$ gives rise to a topology (the spectral or hull-kernel topology) on $\operatorname{Spec} G$, whose closed sets are exactly the subsets $E \subseteq \operatorname{Spec} G$ such that $\mathbb{V} \circ \mathbb{I}(E)=E$. We will shortly see that $\operatorname{Spec} G$ is a spectral space; it is easy to show directly that it is $T_{0}$, whence it carries a specialization order. Specifically, the specialization order $\preceq$ on $\operatorname{Spec} G$ defined by

$$
\mathfrak{p} \preceq \mathfrak{q} \text { if, and only if, } \mathfrak{p} \in \mathbb{V} \circ \mathbb{I}(\mathfrak{q})
$$

coincides with the set-theoretic inclusion $\subseteq$. It is well known [4, 2.4.1 and 10.1.11] that (Spec $G, \preceq$ ) is a root system: each upper set $\uparrow \mathfrak{p}:=\{\mathfrak{q} \in \operatorname{Spec} G \mid \mathfrak{p} \preceq \mathfrak{q}\}$ is linearly ordered. This property is usually called the complete normality of $\operatorname{Spec} G$. It is also known [4, 10.2.2]

\footnotetext{
${ }^{1}$ We recall that congruences of $\ell$-groups (or vector lattices, or $\mathbb{Q}$-vector lattices) are in one-one inclusionpreserving correspondence with kernels of homomorphisms, known as $\ell$-ideals. These can be characterised as the order-convex subalgebras in each of the varieties under consideration.
} 
that $\operatorname{Spec} G$ is a compact space if, and only if, $G$ may be equipped with a (strong order) unit, i.e. there exists $u \in G$ such that for all $g \in G$ we have $n u \geqslant g$ for some integer $n \geqslant 1$. In this case $G$ is called a unital $\ell$-group (or vector lattice, or $\mathbb{Q}$-vector lattice). For the remainder of this section we always assume that $G$ is unital.

It turns out that $\operatorname{Spec} G$ may be regarded as the spectrum of prime ideals of a distributive lattice. Finitely generated congruences on $G$ are the same thing as principal (i.e. singly generated) congruences on $G$. Indeed, writing $\langle T\rangle$ for the congruence on $G$ generated by $T \subseteq G \times G$, we have $\langle(g, 0)\rangle=\langle(|g|, 0)\rangle$, where $|g|:=g \vee-g$ is the absolute value of $g \in G$; and, moreover, $\langle(g, 0)\rangle \cap\langle(h, 0)\rangle=\langle(|g| \wedge|h|, 0)\rangle$ and $\langle(g, 0)\rangle \vee\langle(h, 0)\rangle=\langle(|g| \vee|h|, 0)\rangle$ for all $g, h \in G$. Observe that the top element of Con $G$, namely, the improper congruence $G \times G$, is principal, because $\langle(u, 0)\rangle=G \times G$ for any unit $u \in G$. It follows that $\mathrm{K}(G)$ is a (distributive) sublattice of Con $G$. Writing $\operatorname{Spec}_{\mathrm{DL}} \mathrm{K}(G)$ for the spectrum of prime ideals of $\mathrm{K}(G)$ with the Stone topology, it can now be shown that $\operatorname{Spec}_{\mathrm{DL}} \mathrm{K}(G)$ is homeomorphic to Spec $G$; the homeomorphism takes a prime ideal $P \subseteq \mathrm{K}(G)$ to the congruence $\bigcap P$ on $G$.

By the foregoing, together with Stone duality, we have that Spec $G$ equipped with the spectral topology is a spectral space. The correspondence

$$
\langle(g, 0)\rangle \in \mathrm{K}(G) \quad \longmapsto \quad \operatorname{Spec} G \backslash \mathbb{V}(g)
$$

yields a lattice homomorphism between $\mathrm{K}(G)$ and the lattice of compact open subsets of $\operatorname{Spec} G$.

\section{$3 \quad$ Finitely Presented Structures, and Polyhedra}

For background on polyhedral geometry see [9]. A subset $C$ of finite-dimensional Euclidean space $\mathbb{R}^{n}$ is convex if it contains, together with $p, q \in C$, the entire line segment $\{\lambda p+(1-\lambda) q \mid$ $\lambda \in \mathbb{R}, 0 \leqslant \lambda \leqslant 1\}$. Given a subset $S \subseteq \mathbb{R}^{n}$ of finite-dimensional Euclidean space, its convex hull of $S$, written conv $S$, is the intersection of all convex subsets of $\mathbb{R}^{n}$ that contain it. A polytope in $\mathbb{R}^{n}$ is the convex hull of a finite subset of $\mathbb{R}^{n}$. Polytopes are thus compact and convex. A polyhedron in $\mathbb{R}^{n}$ is any subset that can be written as the union of finitely many polytopes. Polyhedra are thus compact, but not necessarily convex. A polytope is rational if it can be written as the convex hull of a finite subset of $\mathbb{Q}^{n} \subseteq \mathbb{R}^{n}$. A polyhedron is rational if it can be written as the union of finitely many rational polytopes.

For $P \subseteq \mathbb{R}^{n}$ a polyhedron, a continuous map $f: P \rightarrow \mathbb{R}$ is piecewise linear (P.L. for short) if there exist finitely many affine linear functions $l_{1}, \ldots, l_{u}: \mathbb{R}^{n} \rightarrow \mathbb{R}$ such that, for each $p \in P$, we have $f(p)=l_{i_{p}}(p)$ for some $i_{p} \in\{1, \ldots, u\}$. If the $l_{i}$ 's in this definition can be chosen to have rational coefficients (i.e. each $l_{i}$ is the extension to $\mathbb{R}^{n}$ of an affine linear map $\mathbb{Q}^{n} \rightarrow \mathbb{Q}$ ) then $f$ is a rational P.L. map. And if the they can be chosen to have integer coefficients (i.e. each $l_{i}$ is the extension to $\mathbb{R}^{n}$ of a $\mathbb{Z}$-module map $\mathbb{Z}^{n} \rightarrow \mathbb{Z}$ ) then $f$ is a $\mathbb{Z}$-map.

Definition 3.1. For any polyhedron $P \subseteq \mathbb{R}^{n}$, we write $\nabla(P)$ to denote the collection of all P.L. maps $P \rightarrow \mathbb{R}$. Assume further that $P$ is rational. Then we write $\nabla_{\mathbb{Q}}(P)$ the collections of all rational P.L. maps $P \rightarrow \mathbb{R}$, and $\nabla_{\mathbb{Z}}(P)$ for the collection of all $\mathbb{Z}$-maps $P \rightarrow \mathbb{R}$.

Write $1_{P}: P \rightarrow \mathbb{R}$ for the function constantly equal to 1 on $P$. It is an exercise to prove that:

- $\nabla_{\mathbb{Z}}(P)$ is an $\ell$-group having $1_{P}$ as a unit.

- $\nabla_{\mathbb{Q}}(P)$ is a $\mathbb{Q}$-vector lattice having $1_{P}$ as a unit.

- $\nabla(P)$ is a vector lattice having $1_{P}$ as a unit. 
Lemma 3.2. Let $G, R$, and $V$ be a finitely presented $\ell$-group, $\mathbb{Q}$-vector lattice, and vector lattice, respectively. Let $u$ be a unit of $G, R$, or $V$, respectively. Then there exist rational polyhedra $P$ and $Q$, and a polyhedron $T$, such that:

- $(G, u) \cong_{u}\left(\nabla_{\mathbb{Z}}(P), 1_{P}\right)$.

- $(R, u) \cong_{u}\left(\nabla_{\mathbb{Q}}(Q), 1_{Q}\right)$.

- $(V, u) \cong_{u}\left(\nabla(T), 1_{T}\right)$.

Moreover, $P, Q$, and $T$, regarded as topological spaces, are naturally homeomorphic to $\operatorname{Max} G$, $\operatorname{Max} R$, and $\operatorname{Max} V$, respectively.

Remark 3.3. The representation theorem given in the preceding lemma is part of BakerBeynon duality and its variants. See [1, 2, 3]. For MV-algebras see [7].

\section{The Heyting Algebra of Principal Congruences of Finitely Presented Structures}

To prove Theorem 1.1 we relate the spectral space of finitely presented structures with the geometric representation recalled in Section 3. Given a polyhedron $P$, let Sub $P$ denote the collection of all polyhedra contained in $P$. Observe that $\operatorname{Sub} P$ is a distributive lattice under intersections and unions, with top element $P$ and bottom element $\emptyset$. If $P$ is rational, we let $\operatorname{Sub}_{\mathbb{Q}} P$ denote the sublattice of Sub $P$ consisting of the rational polyhedra contained in $P$. Given a lattice $L$ we write $L^{\mathrm{op}}$ to denote the order-dual of $L$ obtained by reversing the order of $L$.

Lemma 4.1. (i) Let $(G, u)$ be a finitely presented $\ell$-group with unit $u$, and let $P$ be a rational polyhedron such that there is a unital isomorphism $\varphi:(G, u) \rightarrow\left(\nabla_{\mathbb{Z}}(P), 1_{P}\right)$ as in Lemma 3.2.

$$
\langle(g, 0)\rangle \in \mathrm{K}(G) \quad \longmapsto \quad \varphi(g)^{-1}(0) \in \operatorname{Sub}_{\mathbb{Q}} P
$$

yields a lattice isomorphism between $\mathrm{K}(G)$ and $\left(\operatorname{Sub}_{\mathbb{Q}} P\right)^{\mathrm{op}}$. Similarly, if $(R, u)$ is a finitely presented $\mathbb{Q}$-vector lattice, there is a lattice isomorphism between $\mathrm{K}(R)$ and $\left(\mathrm{Sub}_{\mathbb{Q}} Q\right)^{\mathrm{op}}$, where $Q$ is a rational polyhedron as in Lemma 3.2.

(ii) Let $(V, u)$ be a finitely presented vector lattice with unit $u$. let $T$ be a polyhedron such that there is a unital isomorphism $\varphi:(V, u) \rightarrow\left(\nabla(T), 1_{T}\right)$ as in Lemma 3.2.

$$
\langle(v, 0)\rangle \in \mathrm{K}(V) \longmapsto \varphi(v)^{-1}(0) \in \operatorname{Sub} T
$$

yields a lattice isomorphism between $\mathrm{K}(V)$ and $(\mathrm{Sub} T)^{\mathrm{op}}$.

Remark 4.2. Observe that, as a consequence of the preceding lemma together with Stone duality, if $P$ is a rational polyhedron then $\operatorname{Spec} \nabla_{\mathbb{Q}}(P)$ is homeomorphic to $\operatorname{Spec} \nabla_{\mathbb{Z}}(P)$, while $\operatorname{Spec} \nabla_{\mathbb{Q}}(P)$ is not homeomorphic to $\operatorname{Spec} \nabla(P)$.

The proof of Theorem 1.1 can now be reduced to the following geometric lemma.

Lemma 4.3. Let $A$ and $B$ be two polyhedra in some Euclidean space $\mathbb{R}^{n}$. Then the topological closure of $A \backslash B$ is a polyhedron of $\mathbb{R}^{n}$. Moreover, if $A$ and $B$ are rational polyhedra, then the topological closure of $A \backslash B$ is a rational polyhedron, too.

The preceding lemma asserts that the lattice Sub $T$ of a polyhedron (or the lattice $\mathrm{Sub}_{\mathbb{Q}} P$ of a rational polyhedron) has the structure of a dual Heyting algebra.Via Lemma 4.1 this translates to the fact that the lattice $\mathrm{K}(G)$ of principal congruences of a finitely presented $\ell$-group (or vector lattice, or $\mathbb{Q}$-vector lattice) $G$ is closed under the Heyting implication of Con $G$. 


\section{Compactness of Spaces of Minimal Primes}

We give two consequences of our main result that will be used in the second part of this abstract. For $L$ a distributive lattice, Speed [10] gave a necessary and sufficient condition for the subspace of minimal primes of $\operatorname{Spec}_{\mathrm{DL}} L$ to be compact. It is elementary to verify that whenever $\operatorname{Spec}_{\mathrm{DL}} L$ is such that its compact open subsets form a Heyting subalgebra of the Heyting algebra of open sets $\mathscr{O}\left(\operatorname{Spec}_{\mathrm{DL}} L\right), L$ satisfies Speed's condition. The converse fails. Recalling that a compact Hausdorff space is Boolean if its clopen sets form a basis for the topology, we have:

Corollary 5.1. Let $G$ be a finitely presented unital $\ell$-group (or unital vector lattice, or unital $\mathbb{Q}$-vector lattice). Then the subspace $\operatorname{Min} G \subseteq \operatorname{Spec} G$ of minimal prime congruences of $G$ is a Boolean space.

Let $G$ be a finitely presented unital $\ell$-group (or unital vector lattice, or unital $\mathbb{Q}$-vector lattice), and let $\mathfrak{m}$ be a maximal congruence of $G$. The germinal congruence at $\mathfrak{m}$ is defined by

$$
\operatorname{germ} \mathfrak{m}:=\bigcap_{\mathfrak{p} \subseteq \mathfrak{m}} \mathfrak{p},
$$

where $\mathfrak{p}$ ranges over prime congruences of $G$. For each $\mathfrak{p} \in \operatorname{Spec} G$, we denote by $\downarrow \mathfrak{p}$ the lower set $\{\mathfrak{q} \in \operatorname{Spec} G \mid \mathfrak{q} \preceq \mathfrak{p}\}$. Arguments similar to the ones proving the preceding corollary yield:

Corollary 5.2. Let $G$ be a finitely presented unital $\ell$-group (or unital vector lattice, or unital $\mathbb{Q}$-vector lattice). Let $\mathfrak{m}$ be any maximal congruence of $G$. Then $\downarrow \mathfrak{m} \subseteq \operatorname{Spec} G$ equipped with the subspace topology is homeomorphic to $\operatorname{Spec}(G /$ germ $\mathfrak{m})$, and is therefore a spectral space. The subspace Min $(\downarrow \mathfrak{m})$ of $\downarrow \mathfrak{m}$ is a Boolean space.

\section{References}

[1] Kirby A. Baker. Free vector lattices. Canad. J. Math., 20:58-66, 1968.

[2] W. Meurig Beynon. Duality theorems for finitely generated vector lattices. Proc. London Math. Soc. (3), 31:114-128, 1975 part 1.

[3] W. Meurig Beynon. Applications of duality in the theory of finitely generated lattice-ordered abelian groups. Canad. J. Math, 29(2):243-254, 1977.

[4] Alain Bigard, Klaus Keimel, and Samuel Wolfenstein. Groupes et anneaux réticulés. Lecture Notes in Mathematics, Vol. 608. Springer-Verlag, Berlin, 1977.

[5] Roberto L. O. Cignoli, Itala M. L. D'Ottaviano, and Daniele Mundici. Algebraic foundations of many-valued reasoning, volume 7 of Trends in Logic-Studia Logica Library. Kluwer Academic Publishers, Dordrecht, 2000.

[6] Peter T. Johnstone. Stone spaces, volume 3 of Cambridge Studies in Advanced Mathematics. Cambridge University Press, Cambridge, 1986. Reprint of the 1982 edition.

[7] Vincenzo Marra and Luca Spada. The Dual Adjunction between MV-algebras and Tychonoff Spaces. Studia Logica, 100(1-2):253-278, 2012. Special issue in memoriam Leo Esakia.

[8] Daniele Mundici. Interpretation of $\mathrm{AF} C^{*}$ algebras in Łukasiewicz sentential calculus. Journal of Functional Analysis, 65:15-63, 1986.

[9] Colin P. Rourke and Brian J. Sanderson. Introduction to piecewise-linear topology. Springer Study Edition. Springer-Verlag, Berlin, 1982. Reprint.

[10] Terence P. Speed. Some remarks on a class of distributive lattices. J. Austral. Math. Soc., 9:289296, 1969. 